\title{
Evolución de la equidad del sistema sanitario brasileño desde las perspectivas del gasto y de la financiación: periodo de 1988 a 2000
}

Evolution of the equity of the brazilian health system from the perspectives of spending and financing: period 1988-2000 Evolução da equidade do sistema sanitário brasileiro desde as perspectivas do gasto e do financiamento: período 1988 a 2000

Marcia Das Mercês Silva Universidad Presbiteriana Mackenzie en São Paulo, Brasil.

E-mail:marciamerces@hotmail.com

\author{
Laura Cabiedes Miragaya \\ Universidad de Oviedo, España. \\ E-mail: Icabie@uniovi.es
}

Fecha de recepción: 22/09/2016 Fecha de aceptación: 20/04/2017
Palabras clave - economía de la salud

- equidad - desigualdad - Brasil

\section{Resumen}

Al inicio de los años 90, el Coeficiente de Gini situaba a Brasil entre los países con peor distribución de la renta y de la riqueza. En 1988, la redemocratización del país culminó con una Constitución Federal que instituyó un sistema sanitario público nacional, de acceso universal. Este estudio ha analizado si el grado de equidad ha evolucionado desde la implantación del Sistema Único de Salud (SUS) en 1988, hasta el año 2000, pues la desigualdad se mantuvo en niveles altos durante este período. Para ello, se ha analizado el grado de equidad del SUS desde la financiación hasta los indicadores del gasto por región. Si bien se han observado marcadas desigualdades en salud entre las regiones, la comparación de los indicadores sanitarios indica que han disminuido las inequidades en el período analizado.

\footnotetext{
Abstract

At the beginning of the $90 \mathrm{~s}$, Brazil was among the countries with the worst income and wealth distribution according to the Gini coefficient. In 1988, the return to democracy of the country culminated in the promulgation of
} 
Keywords

- Health economics

- equity

- inequality

- Brazil

Palavras-chave

- economia da saúde

- equidade

- desigualdade

- Brasil a Federal Constitution which established a national public health system of universal access. This study analyzed whether the degree of equity evolved from the introduction of the Unified Health System in 1988 to 2000 , given that inequality remained at high levels during this period. For this, the degree of equity of the Unified Health System has been analyzed from the perspectives of financing and the spending indicators by region. Despite the fact that there have been noticeable health inequalities between regions, the comparison of health indicators shows that inequalities decreased during the period analyzed.

\section{Resumo}

Ao início dos anos 90, o Coeficiente de Gini situava o Brasil entre os países com pior distribuição da renda e da riqueza. Sendo que em 1988, a redemocratização do país culminou com uma Constituição Federal que tem instituído um sistema sanitário público nacional, de acesso universal. Este estudo tem analisado se 0 grau de equidade tem evolucionado desde a implantação do Sistema Único de Saúde (SUS) em 1988, até 0 ano 2000, pois a desigualdade manteve-se em patamares altos neste período. Para tanto, tem-se analisado o grau de equidade do SUS desde 0 financiamento até os indicadores do gasto por região. Mesmo que se tem observado marcadas desigualdades em saúde entre as regiões, a comparação dos indicadores sanitários indicaram que têm diminuído as iniquidades no período analisado.

\section{El desafío de la}

\section{implantación de un sistema de salud universal en un escenario de marcadas desigualdades socioeconómicas}

A finales de los '80, en Brasil, se ha desarrollado una reforma sanitaria iniciada por parte de varios sectores de la sociedad que, con la apertura política, pudieron manifestar su insatisfacción frente al agotamiento del sistema anterior. La Constitución Federal brasileña, promulgada en 1988, es un marco fundamental de la redemocratización de Brasil. El apartado dedicado a la salud comienza con la frase "La salud es un derecho de todos y un deber del Estado», una conquista social ya que la atención sanitaria no era considerada un derecho social. En cumplimiento a lo que establecía la Constitución Federal de 1988, se creó el Sistema Único de Saúde (SUS, en adelante); un sistema sanitario público nacional, de acceso universal, en paralelo a todo un proceso de descentralización territorial.

Antes de la creación del Sistema Único de Salud, las acciones universales en salud pública en Brasil se resumían a las actividades de promoción y prevención de enfermedades (vacunación, control sanitario de las fronteras) y la asistencia médico-hospitalaria para algunas enfermedades específicas. El Instituto Nacional de Previdencia y Asistencia (INAMPS, en adelante) creado en 1974, por el régimen militar, dentro de la estructura del Ministerio de Previdencia tenía la finalidad de dar asistencia a los trabajadores que contribuyan con la previsión social. En 1990, el INAMPS pasó a componer la estructura del Ministerio de salud y en 1993, tras 3 años de implementación del SUS fue extinto. 
De esta manera, se podía dividir la población en cuanto al acceso a la atención sanitaria entre los que tenían derecho a la salud pública porque estaban asegurados por la previsión social (los trabajadores con empleo formal); los que podían pagar por los servicios privados de salud y los que no poseían derecho alguno.

Sin embargo, aunque la Carta Magna es muy innovadora en lo que se refiere al compromiso del Estado con los derechos sociales, más de una década después, Brasil se situaba entre los países con peor distribución de la renta y de la riqueza, según datos de la CEPAL y Banco Mundial (2002). El desafío de la implantación de un sistema sanitario de acceso universal en este periodo de grande desigualdad socioeconómica es importante para el estudio de la equidad en salud. Brasil ha empezado la década de los 90 con una acentuada desigualdad y según datos del Banco Mundial, en 1990 el país ocupaba el puesto de país más desigual del mundo. Según datos de la Comisión Económica para América Latina (CEPAL, en adelante) el Índice de Gini' ha aumentado, pasando del 0,627 en el año 1990 a 0,640 en 1999 (CEPAL, 2002). No obstante, es preciso señalar que existen contradicciones en los datos referidos al Índice de Gini para Brasil. Con todo, sea cual sea la fuente utilizada, lo que es indiscutible es que el índice alcanza niveles absolutos muy elevados en 1999/2000, evidenciando una distribución de la renta muy desigual (véase la Tabla 1).

Tabla 1. Variaciones en los índices de Gini, según las fuentes

\begin{tabular}{|c|c|c|c|c|c|}
\hline \multicolumn{6}{|c|}{ ÍNDICE DE GINI - BRASIL } \\
\hline AÑ̃O & CEPAL (1) & IPEA (2) & BCO. MUNDIAL (3) & AÑ̃ & IBGE (4) \\
\hline 1990 & 0,627 & 0,616 & 0,604 & 1991 & 0,637 \\
\hline 1999 & 0,640 & 0,596 & 0,589 & 2000 & 0,609 \\
\hline
\end{tabular}

Fuente: 1) CEPAL (2002). 2) IBGE (2003). 3) Banco Mundial (2002). 4) IBGE (2003)
La pobreza y la mala salud están interrelacionadas. El autor Wagstaff destaca que «esta casualidad funciona en los dos sentidos: la pobreza genera mala salud, y la mala salud hace que los pobres sigan siendo pobres» (Wagstaff, 2002:316)

Las desigualdades sanitarias, y muy probablemente también en la utilización de los servicios, reflejan en gran medida las desigualdades con respecto a variables tanto individuales como familiares, tales como la educación, los ingresos, la localización y las características de los hogares. Esto indica que las políticas destinadas a combatir las desigualdades en el sector de la salud deberían dirigirse a reducir las desigualdades tanto en el lado de la oferta (por ejemplo, la calidad y la disponibilidad de servicios de salud), como en el lado de la demanda (ingresos, conocimientos, y especialmente conocimientos relacionados específicamente con la salud, accesibilidad de los servicios de salud, disponibilidad de agua potable segura, saneamiento, etc. (Wagstaff, 2002).

En este contexto de desigualdad social y de construcción de un sistema sanitario público de carácter universal, el tema de la equidad es particularmente relevante. Las marcadas desigualdades interpersonales de la renta en Brasil recomiendan aplicar simultáneamente la dimensión geográfica y la estratificación socioeconómica, pero la escasa disponibilidad de información estadística impide seguir este enfoque. El estudio se ha centrado en la equidad territorial no solamente por esta razón, sino también porque las desigualdades entre las regiones son tan marcadas que se entiende que un mínimo de equidad territorial es condición necesaria, aunque no suficiente, para alcanzar mayor equidad interpersonal.

(1) El Índice de Gini se calcula como la superficie entre una curva de Lorenz y la línea de la igualdad absoluta y varía desde 0 (igualdad perfecta) hasta 1 (desigualdad perfecta). 


\section{Enfoque metodológico para analizar la equidad del sistema sanitario brasileño}

La equidad es un concepto cambiante en la medida en la que cambia la idea de lo que es socialmente justo. Asimismo, dependiendo de los valores dominantes de una organización social derivarán distintas ideas de justicia y distintos principios de distribución. Generalmente, el concepto y los objetivos de equidad que inspiran las políticas sociales no están literalmente mencionados en los textos legislativos, como es el caso de Brasil.

Dependiendo de los principios de distribución dominantes, los sistemas sanitarios pueden ser públicos (marxistas/socialdemócratas) 0 privados (neoliberales). Sin embargo, «en la práctica, en la mayoría de los países la sanidad es financiada y prestada bajo una mezcla de sistemas con rastros de ambas ideologías» (Rodríguez, 1992:58) al identificar los objetivos o conceptos de equidad en sanidad que se derivan de estos principios y teorías, Pereira (1988) hace una importante contribución para el entendimiento de la equidad al enlazar los objetivos de equidad en salud con los principios generales de justicia y con los puntos de vista que mantiene la sociedad de los cuales se derivan (Pereira, 1988). Con base en este estudio, según las teorías de la sociedad y los principios de distribución, los objetivos de equidad pueden ser subdivididos como se ve en el Cuadro 1.

Cuadro 1. Teorías de la sociedad, principios de distribución y objetivos de equidad en política sanitaria

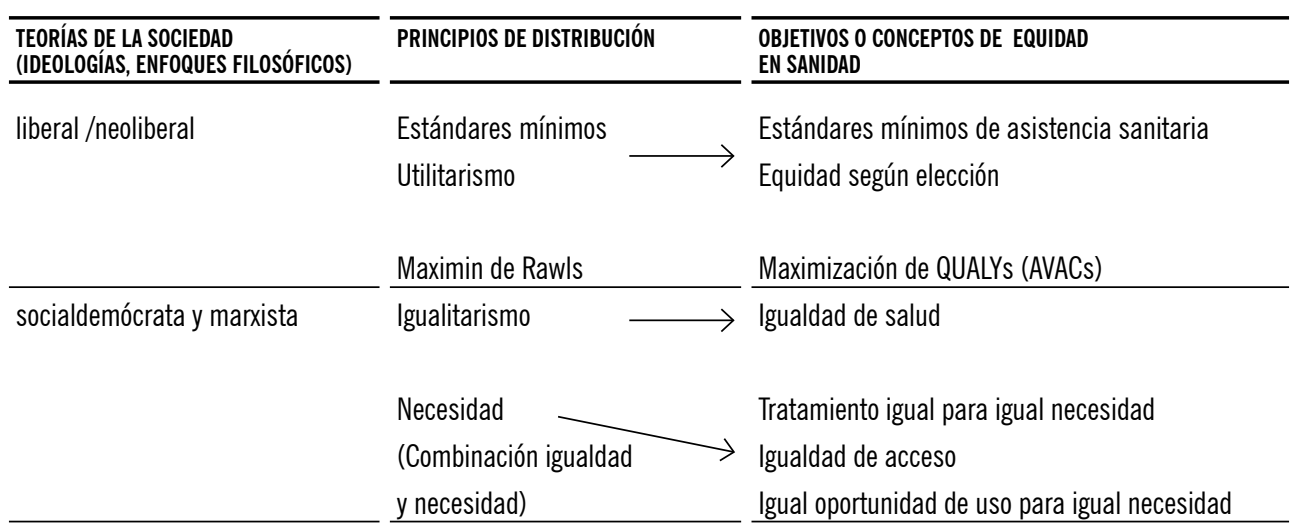

Fuente: Cabiedes (2000) adaptado de Pereira (1988) y Rodríguez (1992).

Derivado del principio neoliberal de estándares mínimos, el objetivo del establecimiento de estándares mínimos de asistencia sanitaria (como por ejemplo el Piso de Asistencia Básica en Brasil), pretende garantizar que nadie se encuentre por debajo de unos «mínimos que repugnarían la conciencia social» (Rodríguez, 1992:56). Como objetivo de disminuir desigualdades en salud, este criterio no es tan atractivo, pues por encima de estos mínimos cada individuo tendrá provisión de asistencia sanitaria de acuerdo con sus condiciones socioeconómicas. Asimismo, existe la dificultad de definir un mínimo digno. Sin embargo, los más pobres pueden tener una atención sanitaria mínima y las desigualdades aunque no disminuyan serían menos injustas, situándose la principal crítica en el hecho de que «... generalmente sus defensores se resisten a fijar de forma explícita esos mínimos» (Rodríguez, 1992:56). 
El utilitarismo es un principio liberal que defiende que el Estado promueva el mayor bienestar posible para el mayor número posible de personas (Albi et ál., 2000:102). De este modo, los criterios que provienen de esta filosofía no ponen el acento en la disminución de desigualdades. «El utilitarismo no tiene en cuenta las características individuales de los individuos comparados ni la distribución interpersonal» (Rodríguez, 1992:58). Por lo tanto constituye un principio poco convincente para fundamentar la equidad.

El principio de distribución Maximin de Rawls es así conocido pues, según la Teoría de Justicia de Rawls (1971), asociada a la teoría del contrato social, los principios de justicia más adecuados serían «aquellos que acordarían libremente los integrantes de una sociedad». Según Rawls, si los individuos desconociesen qué lugar van ocupar en la sociedad y principalmente, si la decisión fuera tomada bajo lo que el autor llama un «velo de ignorancia», que garantizase la imparcialidad de las decisiones, los individuos intentarían protegerse de lo peor (Albi et ál., 2000:104-105). Debido a la posibilidad de verse en una situación desfavorable, se optaría por maximizar el peor resultado (mínimo). Por ello este criterio es conocido como Maximin de Rawls. Al contrario que el utilitarismo, que prioriza la maximización de la utilidad para el mayor número de personas en lugar de la distribución interpersonal, el principio de Maximin pone el acento en la condición individual, en términos del grupo menos aventajado. Parece que este criterio aunque se oriente a mejorar la situación de los menos favorecidos, se despreocupa de qué está ocurriendo con el grupo de los más favorecidos y, más aun, de cómo evoluciona la brecha entre un grupo y otro. Pereira (1988:62) Ilama la atención sobre las dificultades teóricas y prácticas de la aplicación de este principio en sanidad. Plantea la cuestión sobre la definición de los menos aventajados, pregunta si se basaría en términos de su consumo global de bienes primarios, de su salud o en cuanto al nivel de atención sanitaria que reciben.
De acuerdo con el concepto de equidad según elección, las desigualdades en salud ocasionadas por factores que están bajo el control del individuo no pueden ser consideradas inequitativas o injustas. Como por ejemplo fumadores y no fumadores, con las mismas condiciones socioeconómicas y acceso a la misma información. La principal crítica señalada por Rodríguez (1992:59) es la dificultad de definir hasta qué punto ciertos factores están bajo el control de los individuos. Otra crítica es que los individuos deberían estar bien informados (que generalmente no es el caso en el ámbito sanitario) para valorar si una situación deriva o no de la libre elección.

La medida años de vida ajustados según calidad (AVACs), generalmente, es aplicada para evaluar la eficiencia de una política sanitaria, como la variable a maximizar. Pese a ser una medida considerada adecuada para orientar una eficiente asignación de los recursos en salud, este criterio no garantiza una asignación equitativa, pues enfatiza la maximización de los AVACs (criterio de eficiencia) y no la distribución interpersonal (criterio de equidad), excluyendo a los individuos con menor capacidad de beneficiarse del tratamiento (Rodríguez, 1992:59).

De la óptica igualitarista proceden los conceptos de igualdad de salud y los que combinan igualdad con necesidad: tratamiento igual para igual necesidad, igual acceso para igual necesidad e igual oportunidad de uso/acceso ante la misma necesidad. Para Mooney (1994:73), la igualdad de salud como objetivo de equidad es considerada irrealista. Entre otras, la principal crítica es que mientras la atención sanitaria es un bien intercambiable, la salud no lo es y además, sobre ella influyen diversos factores. Así, no cabe pensar en empeorar la salud de unos para mejorar la de otros. A su vez, adoptar sólo medidas en sentido "ascendente», se aproxima más al objetivo de eficiencia que al de equidad. Entre otras razones, Mooney (1994) cuestiona este objetivo, considerando que, aunque todos naciesen con igual salud, serían necesarias condiciones muy estrictas 
para mantener esta igualdad toda la vida, como mantener el mismo estilo de vida, que todos tuviesen las mismas preferencias, ocupaciones y condiciones laborares. Cabiedes (2000:49) aclara que, en la práctica, las políticas sanitarias equitativas operan en el sentido de igualar los recursos o los servicios sanitarios y no la salud, porque la salud es caracterizada "por complejas relaciones de multicausalidad, imposibilidad de intercambiabilidad (entre personas o territorios), dificultades de medida y comparabilidad».

El objetivo de equidad como igualdad de tratamiento para igual necesidad puede ser entendido tanto desde la perspectiva de la equidad horizontal (igual tratamiento ante la misma necesidad) como desde la perspectiva de la equidad vertical (distintos tratamientos ante distinta necesidad). Le Grand (1988:56) advierte de las dificultades de concepción de este enfoque en la práctica. Por ejemplo, ¿el tratamiento está pensado en términos de cantidades de cuidado médico (número de consultas, días de estancia hospitalaria, etc.); gastos en ese cuidado médico (el coste de los recursos) o la valoración subjetiva (valor asignado al tratamiento por los individuos implicados)? Además plantea si por necesidad se entiende la cantidad que los individuos desean o la que un profesional consideraría necesario en cada caso.

Se puede entender la igualdad en acceso para igual necesidad como igualar los costes de oportunidad de acceder a un determinado servicio sanitario, o sea, el coste personal incluyendo desplazamiento y tiempo de espera que un individuo emplearía para recibir un tratamiento médico. Pero en la práctica este objetivo igualitarista de equidad también plantea dificultades. Por ejemplo, medir el acceso es impracticable porque se precisaría un volumen ingente de información. Le Grand (1988:58) plantea cómo se debería medir el «coste personal» ien términos de dinero 0 de privación de la utilidad o satisfacción? En cuanto a las pérdidas debidas al tiempo icómo se debería medir? Otro planteamiento fundamental que hace es si lo importante es el acceso a la atención sanitaria 0 a la salud misma. A su vez, el citado autor considera que no siempre la desigualdad en el acceso es inequitativa, citando el ejemplo de un individuo rico que elige comprar una casa de campo, sabiendo que para acceder a los servicios sanitarios públicos tendrá que utilizar su coche. Mooney (1994:81) entiende que, mientras definir acceso es difícil, la igualdad de acceso, como igual oportunidad de uso o de utilización, es una definición más práctica de la equidad.

Aunque el objetivo de equidad en sanidad no está literalmente recogido en la legislación sanitaria brasileña, sí está recogido el principio de igualdad. En todo caso, al asegurar el acceso universal e igualitario a los servicios sanitarios, el objetivo de equidad implícito en la legislación sanitaria brasileña podría ser interpretado como igualdad en el acceso a la atención sanitaria y, en un intento de traducirlo a términos prácticos, como igual oportunidad de uso 0 acceso ante igual necesidad. Debido a las dificultades que plantea tratar de medir la equidad en términos de acceso, se analiza el grado de la equidad territorial desde la perspectiva tanto del gasto como de la financiación.

La perspectiva de la financiación se apoya básicamente en el estudio del carácter más o menos progresivo del sistema fiscal y de los criterios de asignación de recursos al ámbito sanitario. Para el análisis de la equidad desde la perspectiva del gasto, además de evaluar la dimensión del gasto público, se han recogido algunos indicadores que tratan de aproximar la oportunidad de uso (como los relacionados con la disponibilidad de recursos, la cobertura, la utilización y la calidad) contrastando su variabilidad regional. Asimismo, se ha realizado una mínima aproximación al gasto privado en salud de las familias, que indirectamente mide la incidencia del gasto público, en la medida en la que cabría esperar que las capas más pobres de la población tuviesen que gastar menos en sanidad a partir de la implantación de un sistema sanitario público universal. 


\section{Financiación del sistema sanitario público brasileño}

La Seguridad Social brasileña tiene sus orígenes en los años 30 y abarca la previsión social, la atención sanitaria y la asistencia social (art. 194 de la Constitución). La Constitución Federal, en el artículo 195 establece que la Seguridad Social será financiada por toda la sociedad, de manera directa e indirecta, en los términos de la ley, a través de los recursos procedentes de los presupuestos de los tres niveles del gobierno, las contribuciones sociales de los empresarios y de los empleados, así como la recaudación obtenida del gravamen de los ingresos obtenidos en algunos juegos de azar. Sobre la financiación de la sanidad, la Constitución establece que «el sistema único de salud será financiado en los términos del artículo 195 con recursos del presupuesto de la seguridad social, de la Unión, de los Estados, del Distrito Federal y de los municipios, además de otras fuentes» (art. 198, Párrafo Único de la Constitución).

En definitiva, la sanidad es básicamente financiada de dos maneras; primero con los recursos del presupuesto de la Seguridad Social y, también con recursos de los presupuestos de los tres niveles de gobierno. Los presupuestos de los gobiernos estatales y municipales son financiados con recursos procedentes de la recaudación de impuestos subnacionales (recursos propios) y de transferencias intergubernamentales (del gobierno central a los estados y municipios y de los estados a los municipios) vinculadas (por ejemplo con aplicación exclusiva al sector sanitario o educativo) y sin vinculación.

Sin embargo, el gobierno central a través del Ministerio de Sanidad, responde de la mayor parte de la financiación. Siendo así, para el análisis de la equidad regional en la financiación del SUS, es relevante analizar los impuestos y contribuciones que componen el presupuesto de la Seguridad Social y la estructura fiscal en general, apuntando el carácter más o menos progresivo del sistema.

La carga tributaria brasileña se mantiene por encima del $30 \%$ del PIB y según datos de la Receita Federal de Brasil (2003), órgano responsable por la recaudación, desde 1998 aumentó 6,13 puntos porcentuales y representó en el año 2002 algo más del $35 \%$ del PIB brasileño. Particularmente, en lo que se refiere a la sanidad, de la estructura del sistema fiscal es relevante destacar la Contribución Provisional sobre Movimientos Financieros (CPMF, en adelante) que en el periodo analizado ha sido la principal fuente de financiación del sector sanitario. Es igualmente importante para el sector sanitario resaltar que los gastos privados en sanidad son deducibles del IR. A su vez, la alta carga de los impuestos indirectos incide sobre la compra de medicamentos, lo que suscita distorsiones en la equidad desde la perspectiva del gasto.

Del mismo modo, para la evaluación de la equidad territorial en la financiación de los recursos que componen los presupuestos estatales y municipales, a continuación describimos el grado de descentralización fiscal y los criterios de reparto de las transferencias intergubernamentales.

De acuerdo con la Ley 8080/90 que reglamenta el SUS, los recursos financieros del Sistema Único serán ingresados, en cada esfera de actuación, en una cuenta especial y sus movimientos serán fiscalizados por los Consejos de Salud. En la esfera federal, los recursos originarios del presupuesto de la Seguridad Social y del presupuesto del gobierno federal, además de las otras fuentes, son administrados por el Ministerio de la Salud, a través del Fondo Nacional de Salud. Los estados y municipios administran los recursos de la sanidad a través de los Fondos Sanitarios Estatales y Municipales, respectivamente. ${ }^{2}$ 
El Fondo Nacional de Salud, además de las transferencias «fondo a fondo», transfiere recursos para los convenios y contratos con el sector privado y los pagos a los prestadores de servicios del SUS. Los estados y municipios, además de las transferencias del Fondo Nacional de Salud, aplican parte de sus ingresos propios y de los recursos procedentes de las transferencias del gobierno central (transferencias constitucionales, en adelante). Aunque el artículo 55 del acta de disposiciones constitucionales transitorias establecía que, como mínimo, un $30 \%$ del presupuesto de la seguridad social (excluyendo el seguro de desempleo) debería ser asignado al ámbito sanitario, posteriormente la Ley 8080/90, en su artículo 31, se limita a señalar que el «El presupuesto de la seguridad social destinará al SUS (...) los recursos necesarios para la realización de sus funciones». Según esta ley, las transferencias de recursos hacia los estados y los municipios destinados a la sanidad debían regirse por los siguientes criterios: la mitad del total debía ser repartido en proporción al número de habitantes y el resto, básicamente, según una combinación de criterios, como el perfil demográfico de la región; el perfil epidemiológico de la población; las características de la red sanitaria en la zona y el desarrollo técnico, económico y financiero en el período anterior, entre otros (artículo 35 de la Ley 8080/90). A su vez, el artículo 3 de la Ley 8142/90 establecía que, mientras no fuese regulada la aplicación de los criterios mencionados, sería utilizado exclusivamente el criterio poblacional y que, como mínimo, los municipios recibirían el $70 \%$ de los recursos transferidos, afectando el resto a los estados.

En la práctica, ninguna de las dos leyes fue cumplida íntegramente y la asignación de los recursos federales hacia el SUS se rigió fundamentalmente a través de las Normas Operativas Básicas (NOB, en adelante). ${ }^{3}$ Mozart de Oliveira en la $11^{\text {a }}$ Conferencia Nacional de Saúde (Oliveira,
2001), describe que bajo el argumento de la inviabilidad coyuntural y de las dificultades operativas de regular los criterios previstos en la Ley 8080/90, la NOB 01/91 redefine la lógica de la financiación (y, en consecuencia la organización del SUS), consolidando un sistema de pago al sistema público apoyado en la producción de servicios, en cuyo marco se aplican a los estados y a los municipios las mismas tarifas aplicadas a los proveedores privados. Las transferencias de recursos a los estados y a los municipios prácticamente se limitan a la asistencia ambulatoria y hospitalaria. El resto de las acciones o bien son ejecutadas directamente por el Fondo Nacional de la Salud o se canalizan a través de convenios con los estados, y sólo, marginalmente, con los municipios. Aunque la NOB 01/93 crea diversas modalidades de gestión descentralizada para los municipios (incipiente, parcial y semiplena) y los estados (parcial y semiplena), los avances fueron desiguales y el $75 \%$ de los recursos federales siguió asignándose según el número de procedimientos realizados. Nótese que éste es un método que, al inducir demanda de servicios médico-asistenciales, conlleva cierta dosis de ineficiencia, al tiempo que en modo alguno tiene en cuenta la equidad territorial.

La NOB 01/96 introduce un cambio significativo de los criterios por los que se rige la transferencia de recursos federales: se amplían las transferencias de carácter global «fondo a fondo», directamente desde el Fondo Nacional de la Salud hacia los fondos estatales y municipales, para programas específicos, como el Piso de Atençao Básica (PAB), creado por esta norma para garantizar un valor mínimo per cápita en la financiación de los procedimientos sanitarios básicos. El PAB se transfiere a los municipios habilitados para la gestión plena de la atención básica y se calcula multiplicando el valor per cápita único nacional por la población de cada municipio. Actualmente, además de la parte 
fija originaria, el PAB tiene otra parte variable referida a incentivos para el desarrollo de acciones específicas. Este nuevo enfoque implica a su vez cambios profundos en la gestión del SUS, al permitir a los estados y a los municipios desarrollar su papel como gestores del SUS y no sólo como meros prestadores de servicios. De hecho, a consecuencia de la aplicación de la NOB 01/96 en el año 1998, el porcentaje de recursos federales asignados según el número de procedimientos había caído, ya en 1999 , al 45,9\% (Marques y Mendes, 2001:5).

Entre 1991 y noviembre de 1994 se utilizaron las UCA (Unidades de Cobertura Ambulatoria) para establecer un techo estatal a la atención ambulatoria. Al principio se establecieron seis niveles (reducidos después a cuatro) que pretendían tener en cuenta entre otros aspectos la capacidad instalada; los resultados en términos de calidad y nivel de resolución (de difícil medida); el número de casos atípicos y las series históricas de gasto. En la práctica se aplicaron estos dos últimos, en virtud de los cuales los estados resultaban clasificados con un valor que, multiplicado por el número de habitantes, dejaba definido el techo estatal. A partir de 1994 una comisión tripartita pacta el techo financiero global para cada estado (techo del que el Ministerio de la Sanidad precisa partir para deducir la cantidad transferida a los municipios en gestión semiplena). De nuevo, en la práctica, se utiliza una serie de gastos históricos que implícitamente refleja la capacidad instalada y la complejidad, de modo que a pesar de los cambios más recientes, a la larga la fijación de techos financieros conduce a que los territorios que tradicionalmente concentran más servicios tengan mayor capacidad de captar recursos (Oliveira, 2001).

Aunque este trabajo trata del periodo pos implantación del SUS hasta el año 2000, es importante señalar que el modelo anterior al SUS, ha profundizado las desigualdades en la financiación del sistema. Souza (2002) observa que una vez que el INAMPS aseguraba la atención a los trabajadores con empleo formal, cuanto más desarrollada la economía de una provincia, mayor presencia de relaciones formales de trabajo y mayor era el número de beneficiarios y en consecuencia, mayor la necesidad de recursos para garantizar la asistencia a esta población. El autor resalta que, con eso, el INAMPS aplicaba más recursos en los estados de las regiones sur y sudeste y en mayor proporción en los municipios de mayor porte (Cuadro 2).

Cuadro 2. Desigualdades Regionales en la Asistencia Médica en Brasil, 1986

\begin{tabular}{|c|c|c|}
\hline REGIÕES & $\begin{array}{r}\text { GASTO REGIÃo / } \\
\text { TOTAL DO PAÍs (\%) }\end{array}$ & $\begin{array}{l}\text { POPULAÇÃO REGIĨAO / } \\
\text { TOTAL DO PÁIS (\%) } \\
\end{array}$ \\
\hline Norte & 2,27 & 5,48 \\
\hline Nordeste & 18,1 & 28,82 \\
\hline Sudeste & 59,28 & 43,79 \\
\hline Sul & 15,14 & 15,12 \\
\hline Centro-0este & 5,02 & 6,78 \\
\hline Brasil & 99,81 & 99,99 \\
\hline
\end{tabular}

Fuente: INAMPS DIS. 1987 en Souza (2002).

\section{Algunos indicadores de la equidad relacionados con el gasto sanitario}

En el año 2000, el gasto sanitario total de Brasil (público y privado) fue de 631 dólares per cápita en PPA. En este mismo año, el gasto sanitario público representa sólo el 40 \% del gasto sanitario total. En los países desarrollados donde predomina el enfoque ideológico socialdemócrata, con sistemas nacionales de salud, el gasto público responde de la mayor parte del gasto sanitario total, como Portugal y España, contraponiéndose a los sistemas de países que siguen un enfoque más liberal, como Estados Unidos, donde predomina el sistema privado.

Respecto de la distribución del gasto sanitario público por regiones, resalta que casi el $46 \%$ del 
gasto sanitario público total fue destinado a la región Sudeste en el año 2000 (véase el Cuadro 3), región que concentra el 42,65 \% de la población. Desde la perspectiva del gasto per cápita, el nivel alcanzado en esta región (215,7 reales) es más del $50 \%$ mayor que el gasto correspondiente a la región Nordeste. La región Nordeste, que es la más pobre y presenta los peores indicadores sanitarios, es también la región más dependiente de los recursos federales (el $67 \%$ ). Considerando que la renta per cápita de la región Sudeste es significativamente más alta, esta situación es aún más preocupante desde el punto de vista de la equidad regional.

Cuadro 3. Estimación del gasto sanitario público por regiones y por habitante, año 2000

\begin{tabular}{|c|c|c|c|c|c|c|c|c|c|}
\hline $\begin{array}{l}\text { NIVELES DE } \\
\text { GOBIERNO }\end{array}$ & $\overline{\text { FEDERAL }}$ & & ESTATAL & & MUNICIPAL & & TOTAL & & \\
\hline REGIONES & $\begin{array}{l}\text { \% DEL } \\
\text { GASTO }\end{array}$ & $\begin{array}{r}\text { GASTO POR } \\
\text { HAB. }\end{array}$ & $\begin{array}{l}\text { \% DEL } \\
\text { GASTO }\end{array}$ & $\begin{array}{r}\text { GASTO } \\
\text { POR HAB. }\end{array}$ & $\begin{array}{l}\text { \% DEL } \\
\text { GASTO }\end{array}$ & $\begin{array}{r}\text { GASTO } \\
\text { POR HAB. }\end{array}$ & $\begin{array}{r}\text { GASTO (EN } \\
\text { MLES DE REALES } \\
\text { CORRIENTES) }\end{array}$ & $\begin{array}{r}\% \text { GASTO } \\
\text { TOTAL POR } \\
\text { REGION }\end{array}$ & $\begin{array}{r}\text { GASTO } \\
\text { POR HAB. }\end{array}$ \\
\hline BRASIL & 59,8 & 119,9 & 18,6 & 37,2 & 21,6 & 43,8 & $34.012 .089,0$ & 100,0 & 200,8 \\
\hline Norte & 53,2 & 99,7 & 34,0 & 63,7 & 12,9 & 24,1 & $2.418 .444,0$ & 7,1 & 187,5 \\
\hline Nordeste & 66,9 & 96,0 & 18,4 & 26,4 & 14,7 & 21,0 & $6.846 .035,0$ & 20,1 & 143,4 \\
\hline Sudeste & 51,7 & 111,5 & 19,1 & 41,1 & 29,2 & 63,1 & $15.618 .167,0$ & 45,9 & 215,7 \\
\hline Sur & 59,6 & 104,5 & 16,3 & 28,6 & 24,0 & 42,1 & 4.399.368,0 & 12,9 & 175,2 \\
\hline Centro-0este & 56,6 & 106,0 & 24,6 & 46,1 & 18,7 & 42,6 & $2.177 .569,0$ & 6,4 & 194,6 \\
\hline $\begin{array}{l}\text { Datos no } \\
\text { desagregados } \\
\text { (2) }\end{array}$ & - & 517,6 & 112,4 & 205,9 & 99,5 & 192,9 & $2.552 .505,0$ & 7,5 & 15,0 \\
\hline
\end{tabular}

Fuente: Ministerio da Saúde (2003)

A efectos de realizar una aproximación al análisis de la equidad del sistema sanitario brasileño desde el punto de vista del gasto, se eligió algunos indicadores utilizados habitualmente para evaluar la evolución de la equidad geográfica, referidos a las cinco regiones brasileñas, clasificados en cinco grupos: indicadores de recursos disponibles, gasto, utilización, calidad y cobertura. Obviamente, su aplicación a niveles administrativos inferiores arrojaría un mayor grado de dispersión. Aunque existe información a nivel estatal para muchos de ellos, este enfoque sobrepasaría las dimensiones propias de un trabajo como el aquí presentado. Por otra parte, se persigue un análisis conjunto, más que individualizado, de los indicadores, dando cabida a una acepción amplia del concepto de equidad. Incluso en algunos casos podría ser discutible su empleo como tales, por ejemplo el nivel del gasto por territorios no tiene por qué responder a un enfoque de equidad según necesidad, pero en Brasil las diferencias de partida son tan marcadas que entendemos que una disminución de la brecha que separa las regiones más pobres (Nordeste y Norte) de las más ricas (Sudeste, Sur y Centro-0este), puede ser casi directamente interpretada como una mejora en los niveles de equidad.

Partiendo de las marcadas desigualdades existentes entre las regiones de Brasil, utilizaremos en una primera aproximación el factor de variación como indicador de la variabilidad territorial, 
definido como el cociente entre el valor máximo y el mínimo alcanzado para cada año. No se ha podido analizar el mismo período de tiempo para todos los indicadores, como habría sido deseable. De todas maneras, cuando ha sido posible, se han elegido datos de principios de la década de los '90, momento de la implantación del SUS. Cuando procede comparar la evolución del factor de variación, se ha apuntado la tendencia positiva o negativa, desde el punto de vista de la equidad, entendiendo que una disminución del factor se relaciona con una mejora de la equidad y viceversa. Es preciso reconocer que no se han explotado totalmente las vías de análisis de los datos recogidos. No obstante, se estima que con el análisis presentado se alcanzan los principales objetivos de este trabajo. Además, existen importantes limitaciones en cuanto a la disponibilidad $\mathrm{y} / 0$ calidad de los datos, como las verificadas en los datos de cobertura. Si bien se ha de tener en cuenta la existencia de este tipo de problemas, se estimó conveniente no perder estas informaciones y reunirlas bajo la perspectiva del gasto, con vistas a una evaluación global de los resultados del SUS.

Como se puede observar en el Cuadro 4, existe una importante concentración de recursos humanos en las regiones más ricas, pero la variabilidad territorial disminuyó entre 1990 y 2000 . A modo de ejemplo, aunque en la región Sudeste en el año 2000 existían más del doble de médicos por 1000 habitantes $(2,57)$ que en las regiones Norte y Nordeste (1,02 y 1,13 , respectivamente), esta desigualdad es menor respecto de la registrada en el año 1990, año para el que el factor de variación ascendía a 3,62.

Respecto de los recursos físicos es preciso destacar que en el ámbito de los servicios especializados, el SUS depende considerablemente del sector privado; a modo de ejemplo, el $80 \%$ de los hospitales que prestan servicios al sistema público es de carácter privado. Baste señalar las siguientes cifras referidas a mamógrafos y equipamientos de hemodiálisis: de los 2149 mamógrafos disponibles en el país, el $75 \%$ se concentra en las regiones Sur y Sudeste. El 92,6\% pertenece a establecimientos privados y poco más del $7 \%$ está en instituciones públicas, aunque el 41,6\% está a disposición del sistema público. Más de la mitad de los equipamientos de hemodiálisis se concentra en la región Sudeste frente a un $20 \%$ a disposición de establecimientos localizados en las regiones Norte y Nordeste (OPAS, 2001: 13). La distribución territorial de los recursos de alta tecnología es pues bastante desproporcionada, con acentuada concentración en las regiones ricas. Por ejemplo, aunque la región Sudeste abriga el 42,65 $\%$ de la población, la disponibilidad relativa para la mayoría de los recursos sobrepasa la cota del 50 \%. Por ejemplo en el año 1999, había uno equipo de hemodiálisis por 10000 habitantes en la región Norte, mientras que en la región Sudeste la disponibilidad ascendía a 6,1 equipos por 10000 habitantes (véase el Cuadro 6).

En cuanto al número de camas se ha verificado un cierto equilibrio en la oferta por regiones a partir de 1997, si bien la mayoría de las camas de las que dispone el SUS es de propiedad privada, sobre todo en las regiones ricas. La variabilidad en el número de unidades ambulatorias por 10.000 habitantes ha aumentado. En 1999 las regiones más pobres concentraban más establecimientos de este tipo que la región Sudeste. Aun así, la región Sur es la que concentra más unidades ambulatorias (el doble que la región Sudeste). No obstante, respecto de este dato en particular, las conclusiones precisan un mínimo de cautela, debido a que existen otros tipos de establecimientos $y / 0$ dispositivos que, bajo otras denominaciones, prestan servicios de atención básica.

Con respecto al gasto medio hospitalario (total del gasto dividido por el número de procedimientos), aunque el gasto fue 1,72 veces mayor en la región Sudeste que en la Norte en el año 2000, se verificó una tendencia positiva, ya que esta relación ascendía a 2,04 en 1995. Para el gasto medio en atención ambulatoria se verifica un pequeño 
aumento de la desigualdad entre el peor y el mejor indicador para los años correspondientes, pasando el factor de variación de 1,40 en 1996 a 1,49 en el año 2000. Sin embargo, de acuerdo con los resultados obtenidos en el indicador referido a consultas médicas, cabría esperar una disminución de la variabilidad territorial del gasto ambulatorio. Posiblemente, el hecho de que el indicador de gasto se refiera a gasto por procedimiento podría explicar la aparente contradicción de los datos, ya que nada impide que los costes por procedimiento varíen entre regiones. Así, el hecho de que en consultas por habitante haya disminuido la variabilidad territorial, parece indicar que lo que varía entre regiones es el factor "precio», en el que a su vez podría estar influyendo el componente tecnológico. El número de ingresos por 100 habitantes, en el año 2000, es mayor en las regiones Norte y Nordeste que en la región Sudeste, alcanzando el mayor valor en la región Sur. La variabilidad territorial de este indicador ha disminuido entre los años 1991 y 2000, sobre todo por la disminución del número de ingresos en las regiones más ricas. Además, es preciso ser muy cauteloso en su análisis, porque un elevado número de ingresos puede estar reflejando carencias en el nivel de atención básica. Por su parte, entre 1987 y 2000 aumentó el número de consultas en todas las regiones, pero mucho más en las más pobres.
Con respecto a los indicadores seleccionados para evaluar la calidad, ha aumentado ligeramente la desigualdad territorial en cuanto al porcentaje de gestantes con 60 más consultas, pero es necesario tener en cuenta que sólo se comparan dos años consecutivos. Por otro lado, se observa una disminución de la variabilidad territorial en términos de tasas de mortalidad por causas mal definidas, destacando una fuerte caída de la tasa en la región Nordeste. Nótese que en este caso los datos permiten contrastar años suficientemente distanciados (1990 y 1998).

En cuanto a los datos sobre la cobertura, datos del Ministerio de Salud (2003) apuntan que en el año 2001 el sector público respondía del $96 \%$ de la atención básica y del 57 \% y 73 \% de los procedimientos de media y alta complejidad, respectivamente. No obstante, aunque la cobertura sanitaria primaria, entendida como la asistencia ambulatoria, sea muy elevada, la cobertura farmacéutica, que es uno de los pilares de la asistencia básica, sigue siendo muy reducida. La asistencia básica puede verse seriamente comprometida debido a que Brasil presenta altos niveles de pobreza y muchas personas, aunque reciban atención sanitaria primaria, no pueden hacer frente al tratamiento con medicamentos, problema que puede ser agravado por la presencia de carencias nutricionales. 
Cuadro 4. Indicadores del grado de equidad geográfica del sistema sanitario brasileño (por regiones)

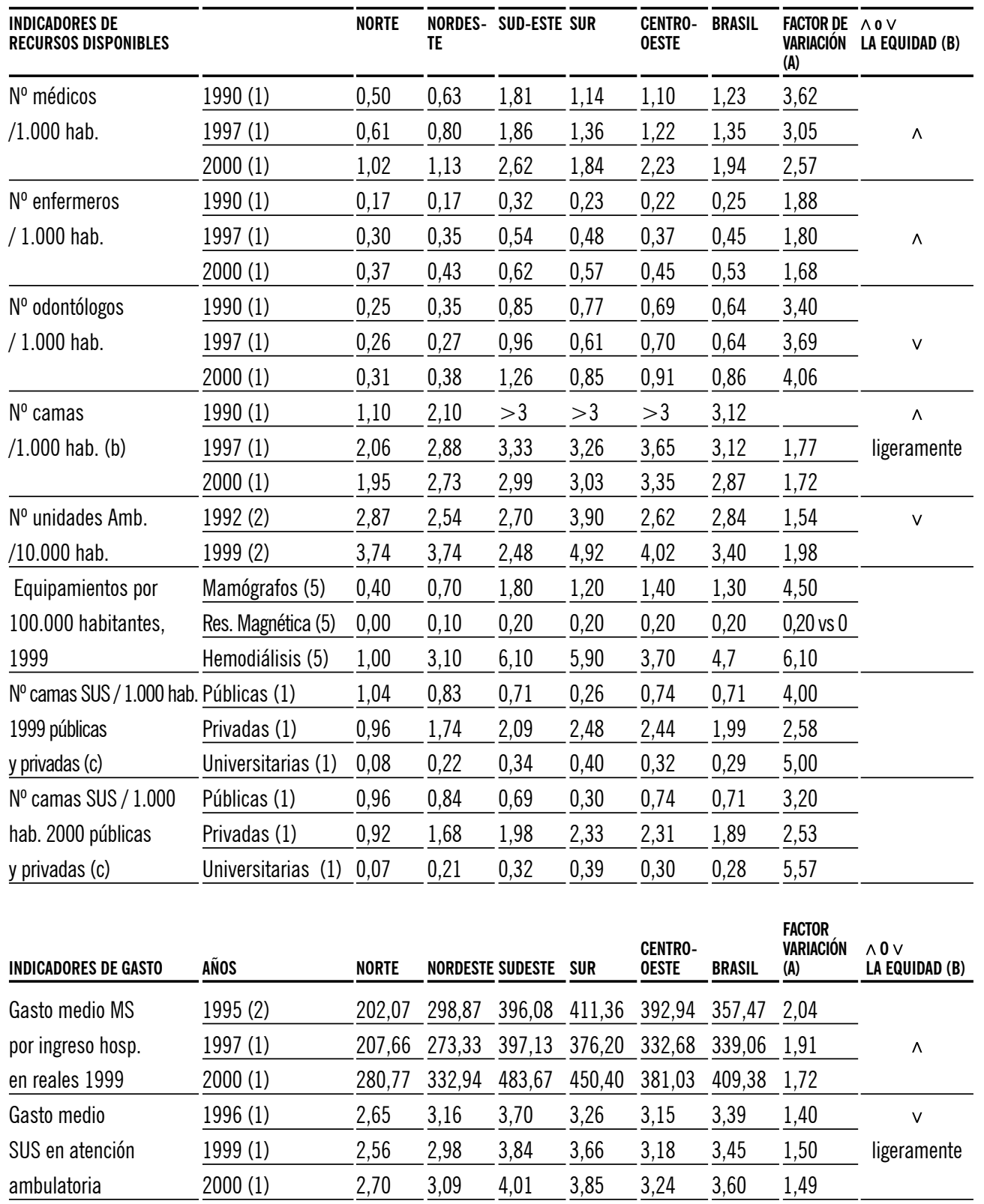

[Cont. pág sgte.] 


\begin{tabular}{|c|c|c|c|c|c|c|c|c|c|}
\hline $\begin{array}{l}\text { INDICADORES DE } \\
\text { UTILIZACIÓN [COnt.] } \\
\end{array}$ & AÑNOS & NORTE & NORDESTI & E SUDESTE & SUR & $\begin{array}{l}\text { CENTRO- } \\
\text { OESTE }\end{array}$ & BRASIL & $\begin{array}{l}\text { FACTOR DE } \\
\text { VARIACIÓN (A) }\end{array}$ & $\begin{array}{l}\wedge 0 \vee \\
\text { LA EQUIDAD (B) }\end{array}$ \\
\hline Consultas SUS & $1987(3)$ & $-0,88$ & $-1,09$ & $-2,21$ & 1,76 & $-1,23$ & $-1,67$ & $-2,51$ & \multirow{3}{*}{$\wedge$} \\
\hline \multirow[t]{2}{*}{ por habitante } & $1997(1)$ & 1,44 & 2,10 & 2,63 & 2,02 & 2,12 & 2,26 & 1,83 & \\
\hline & $2000(1)$ & 1,54 & 2,10 & 2,69 & 2,23 & 2,08 & 2,33 & 1,75 & \\
\hline Ingresos SUS por & $\underline{1991(2)}$ & $-5,64$ & $-8,86$ & $-9,30$ & 10,83 & $-10,66$ & $-9,24$ & 1,92 & \multirow{3}{*}{$\wedge$} \\
\hline \multirow[t]{2}{*}{100 habitantes } & $\underline{1997(1)}$ & $-7,01$ & $-7,54$ & $-6,47$ & $-7,98$ & 7,16 & $-7,37$ & $-1,23$ & \\
\hline & $-2000(1)$ & $-7,15$ & $-7,56$ & $-6,24$ & 7,92 & 7,71 & $-7,03$ & 1,27 & \\
\hline $\begin{array}{l}\text { INDICADORES } \\
\text { DE CALIDAD } \\
\end{array}$ & AÑNOS & NORTE & NORDEST & E SUDESTE & SUR & $\begin{array}{l}\text { CENTRO- } \\
\text { OESTE } \\
\end{array}$ & BRASIL & $\begin{array}{l}\text { FACTOR DE } \\
\text { VARIACIÓN (A) }\end{array}$ & $\begin{array}{l}\wedge 0 \vee \\
\text { LA EQUIDAD (B) }\end{array}$ \\
\hline \% gestantes con 6 ó & $1998(1)$ & 38,02 & 38,83 & $-56,26$ & 53,88 & 55,34 & 49,48 & 1,48 & \multirow[t]{2}{*}{$\mathrm{v}$} \\
\hline más consultas & $1999(1)$ & $-34,21$ & $-45,26$ & $-56,65$ & $-54,04$ & $-51,54$ & $-50,69$ & 1,66 & \\
\hline Tasas mortalidad por & $1990(2)$ & 29,02 & $-42,14$ & $-8,71$ & 12,02 & 12,38 & $-18,17$ & 4,84 & \multirow[t]{2}{*}{$\wedge$} \\
\hline causas mal definidas & $-1998(2)$ & $-24,25$ & $-29,82$ & $-9,95$ & 7,68 & $-10,63$ & $-14,92$ & $-3,88$ & \\
\hline \multicolumn{2}{|c|}{ Hospitales acreditados / 2000 (2) } & 0,32 & 2,16 & 0,37 & 0,97 & 1,92 & 1,07 & 6,75 & \\
\hline 1 millón hab. & & & & & & & & & \\
\hline
\end{tabular}

(a) Factor de variación = cociente entre el valor máximo y el mínimo para cada año.

(b) Se considera que aumenta la equidad si el factor de variación disminuye entre el primer y el último año de la serie estudiada y viceversa, con la excepción del indicador $n^{0}$ de camas/1.000 habitantes para el que se comparan los años de 1997 y 2000, ya que la indefinición de los datos correspondientes a 1990 impide considerar este año.

(c) Observación: las regiones Sudeste y Sur abrigan importantes hospitales públicos universitarios.

Fuentes: (1) Ministerio da Saúde (2003). (2) Nunes, Silva Santos, Barradas Barata y Magalhães Vianna (2001). (3) OPAS (2001) (4) Ministerio da Saúde (2003). (5) Ministerio da Saúde (2003).

Los gastos de sanidad de las familias reflejan en gran medida la capacidad del servicio público de cubrir las necesidades de los ciudadanos y complementa de manera indirecta el análisis de la evolución del grado de equidad del gasto público. Medici (2002:27), contribuye a este enfoque, evidenciando la regresividad de los gastos en sanidad de las familias brasileñas. Comparando los gastos familiares en salud de los años de 1987 y 1996, por estratos de renta, verifica que la participación de los gastos en salud aumentó proporcionalmente más en las familias más pobres, aumentando en un $52 \%$ en los primeros tramos de renta (hasta dos sueldos mínimos) y el $15 \%$ la en el último tramo (más de 30 sueldos mínimos).
Desde el punto de vista regional, Medici (2002:13) verifica que los gastos en seguros sanitarios aumentaron más, en relación con los gastos sanitarios totales de las familias, en las regiones metropolitanas del Norte y Nordeste, lo que según el autor puede ser debido a que estos gastos tienen mayor peso en los ingresos familiares de las familias de esta región, que son más bajos y también porque la oferta de servicios públicos en estas zonas puede ser menor y menos diversificada. En la región Sudeste la excepción es São Paulo, que ha aumentado en el mismo nivel de las ciudades del Norte y Nordeste, lo que puede ser explicado por la gran concentración de oferta de servicios sanitarios privados en la región. 


\section{El impuesto sobre la renta y el peso del sector privado en Brasil}

Es relevante dejar indicado que la compra de servicios sanitarios privados es deducible en el impuesto sobre la renta, lo que distorsiona enormemente la equidad del sistema. Entre otras razones, porque las familias que hacen la declaración de la renta y en consecuencia pueden deducir sus gastos en sanidad privada, son las de renta media y alta, y las familias de renta inferior al nivel exigido para la declaración de la renta no recibirán este beneficio fiscal.

Debido al importante peso del sector privado, conviene no perder de vista el nivel del gasto sanitario privado, con el objetivo de evaluar hasta qué punto el SUS cumple el objetivo de disminuir el gasto sanitario de las familias, principalmente de baja renta, lo que indirectamente complementa el análisis de la equidad en el gasto sanitario público.

\section{Algunos resultados y conclusiones}

En este trabajo se realizó un análisis de la evolución del grado de equidad del sistema sanitario público brasileño, desde su creación hasta el año 2000. Del análisis de los datos recogidos se desprende que el SUS, desde su concepción, ha logrado mejorar los indicadores sanitarios para el conjunto de Brasil, principalmente los correspondientes a las regiones más pobres, que partían de niveles muy alejados de los correspondientes a las zonas más favorecidas.

Desde la perspectiva de la financiación, se observó que la estructura del sistema es compleja y aun hay mucho margen de maniobra para contribuir a mejorar la equidad geográfica, e interpersonal, del sistema sanitario brasileño. Desde esta perspectiva, se deberían hacer mayores esfuerzos en dos sentidos: en primer lugar, imprimiendo mayor progresividad al sistema fiscal en general, lo que permitiría disponer de más recursos para la promoción de políticas sociales y, en particular, para la articulación de un verdadero sistema universal de salud. Además, las mejoras en otras políticas sociales (como educación o vivienda) seguramente tendrían un efecto positivo en la salud de los más desfavorecidos. La reciente aprobada Propuesta de Enmienda Constitucional que crea un techo de gasto público por los próximos 20 años, la PEC 241, no es tratada en este artículo, pero tiene preocupado en cuanto a los avances en las inversiones en salud.

Los indicadores de recursos, gasto, utilización y calidad muestran una elevada variabilidad territorial, pero inferior, en términos generales, a la existente a principios de los años 90 . Se supone (ya que no hemos manejado datos desagregados) que los diversos programas sanitarios e intersectoriales tuvieron un impacto positivo en estos resultados, puesto que la mayoría de ellos están orientados a las regiones y a las capas de la población más pobres.

Cabe observar que la implantación del SUS al establecer el derecho social a la atención sanitaria a todos los brasileños, diferente del modelo anterior, ha atribuido ciudadanía a las personas que antes no tenían derecho alguno. Asimismo, respecto de la financiación sanitaria el SUS ha impartido cierta justicia social, comparado con el modelo anterior que asignaba mayores recursos a los municipios más ricos al debido a la vinculación de derecho a los trabajadores formales. Se entiende, sin embargo, que se deberían hacer mayores esfuerzos mejorando los criterios de reparto, de tal modo que los recursos se fuesen asignando cada vez en mayor medida por criterios de necesidad y no tanto por las inercias del pasado, que favorecen un statu quo poco equitativo y que la PEC 241 todavía no apunta claramente este cambio de dirección.

Por último, se entiende que la creación del SUS $y$ todos sus programas puntuales en regiones con peor acceso, así como la distribución de medicamentos que ocurre actualmente, tanto de los más baratos y principalmente de alto coste, es indiscutiblemente un avance en el bien estar del brasileño, en las últimas décadas a un modelo anterior, 
todavía reciente, que grande parte de la población actual, ha sido afectada, hasta inicio de los ' 90. Así como, reconocer los esfuerzos de numerosos sectores de la sociedad brasileña, de los profesionales del sector sanitario y de la población en general, principalmente los que dependen solamente del sistema público de salud, que siguen luchando para garantizar la conquista del derecho que tienen todos los ciudadanos a una vida saludable, en el sentido más amplio.

\section{Referencias bibliográficas}

- Albi, E., González-Páramo, J.M., y Zubiri, I. (2000). Economía Pública I. Fundamentos, Presupuesto y Gasto, Aspectos Macroeconómicos. BarceIona: Ariel.

- Banco Mundial. (2002). Más Allá Crecimiento Económico. Programa de Educación para el Desarrollo. Instituto del Banco Mundial.

- Constituição da República Federativa do Brasil. Senado Federal, Brasília, DF, 05 de outubro de 1988. Recuperado en julio 2017 de http:// www.planalto.gov.br/ccivil_03/constituicao/constituição.htm.

- Cabiedes, L. (2000). La Financiación del Sistema Sanitario. En Gobierno del Principado de Asturias, Libro Blanco de los Servicios Sanitarios Asturianos (pp.177-216). España: Consejería de Salud y Servicios Sanitarios del Principado de Asturias.

- CEPAL (2002). Anuario Estadístico de América Latina y Caribe 2001. Chile: Naciones Unidas, Comisión Económica para América Latina y el Caribe. Recuperado en Julio 2017 de http://repositorio.cepal. org/bitstream/handle/11362/915/1/ S0111982_mu.pdf
- Enmienda Constitucional $\mathrm{N}^{0}$ 91. Artigo 55, Ato das Disposições Constitucionais Transitórias. Senado Federal. Brasília, DF, 18 de Febrero de 2016. Recuperado en julio 2017 de https://www.senado. gov.br/atividade/const/con1988/ ADC1988_18.02.2016/art_55_.asp

- Instituto Brasileiro de Geografia e Estatística (IBGE). (2003). Censo demográfico 2000. Recuperado en Julio 2017 de http://www.ibge.gov.br/ - Le Grand, J. (1988). Equidad, Salud y Atención Sanitaria. Madrid: Asociación de Economía de la Salud.

- Ley Nro 8080. Lei Orgânica da Saúde L.0.D. Ministério da Saúde, Brasília, Brasil, 19 de setembro de 1990.

- Ley Nro 8142. Lei Orgânica da Saúde L.0.D. Ministério da Saúde, Brasilia, Brasil, 28 de dezembro de 1990.

- Marques, R.M., y Mendes, Á. (2001). 0 Financiamento da Atenção à Saúde no Brasil, Conselho Nacional de Saúde. Saúde Debate, 38(103), 900-919.

- Medici, A.C. (2002). Los gastos en salud en las familias de Brasil: algunas evidencias de su carácter regresivo. Serie de informes técnicos del Departamento de Desarrollo Sostenible. Banco Interamericano de Desarrollo. Recuperado en Julio 2017 de https://publications.iadb.org/ handle/11319/4700

- Ministerio da Saúde (2003). Anuario Estatístico de Saúde do Brasil 2001. Recuperado en Julio 2017 de http://ses.sp.bvs.br/lis/resource/16959\#.WW6AQYQ1_IU

- Mooney, G. (1994). Key Issues in Health Economics. New York and London: Harvester Wheatsheaf.

- Nunes, A., Silva Santos, J.R., Barradas Barata, R., y Magalhães Vianna, S. (2001). Medindo as desigualdades em saúde no Brasil: Uma proposta de monitoramento. Brasília: OPAS (Organización Panamericana de la Salud) e IPEA (Instituto de Pesquisa Econômica Aplicada).

- Oliveira Júnior, M.d. (2001). Critérios para repasse dos recursos federais. Papel redistributivo do Goberno Federal e Estadual no financiamento da saúde. $11^{\text {a }}$ Conferencia Nacional de Saúde, Conselho Nacional de Saúde, Brasil. 
- Organización Panamericana de Salud (OPAS) (2001). 0 perfil do sistema de serviços de saúdeBrasil (2da, edición). Programa de Organização y Gestão dos Sistemas e Serviços de Saúde. Washington, D.C.: OPAS

- Pereira, J. (1988). La interpretación económica de equidad en la salud y atenciones sanitarias. En Asociación de Economía de la Salud, Salud y Equidad, (pp. 31-51). Madrid: Asociación de Economía de la Salud.

- Rawls, J. ([1971] 2000). Teoría de la justicia. México, Fondo de Cultura Económica.

- Resolución Nro. 273. Norma Operacional Básica № 01/91. Ministério da Saúde/INAMPS, Brasil, 17 de julho de 1991. Recuperado en julio 2017 de http://siops.datasus.gov.br/Documentaca0/olu\%C3\%A7\%C3\%A30\%20 258_07_01_1991.pdf

- Resolución Nro. 545. Norma Operacional Básica, NOB - SUS N 01/93. Ministério da Saúde, Brasil, 20 de maio de 1993. Recuperado en julio 2017 de http://bvsms.saude.gov.br/bvs/saudelegis/gm/1993/ prt0545_20_05_1993.html

- Resolución Nro. 2203. Norma Operacional Básica, NOB - SUS N 01/96. Ministério da Saúde, Brasil, 5 de novembro de 1996. Recuperado en julio 2017 de http://bvsms.saude.gov.br/bvs/saudelegis/gm/1996/ prt2203_05_11_1996.html

- Rodríguez, M. (1992). Corrientes económicas y criterios de equidad en Sanidad. Revista de Economía, 12, 55-60.

- Secretaria da Receita Federal. (2003). Estudos Tributários. Recuperado en Julio 2017 de http://www. receita.fazenda.gov.br/.

- Souza, R.R.d. (2002). Construindo o SUS: a lógica do financiamento e o processo de divisão de responsabilidades entre as esferas de governo. Rio de Janeiro: Instituto de Medicina Social da UERJ.

- Wagstaff, A. (2002). Pobreza y desigualdades en el sector de la salud. Revista Panamericana de Salud Publica [online], 11(5-6), 316-326. http://dx.doi.org/10.1590/S102049892002000500007.

\section{Registro bibliográfico}

Das Mercês Silva, M., y Cabiedes Miragaya, L. (2017). Evolución de la equidad del sistema sanitario brasileño desde las perspectivas del gasto y de la financiación: periodo 1988 a 2000. Revista Ciencias Económicas, 14(01), 9-25. 